\title{
Musculoskeletal Pain Due to Wheelchair Use: A Systematic Review and Meta-Analysis
}

\author{
Andreas Liampas · Panayiota Neophytou · Maria Sokratous • \\ Giustino Varrassi · Christiana Ioannou • Georgios M. Hadjigeorgiou • \\ Panagiotis Zis (D)
}

Received: May 25, 2021 / Accepted: July 12, 2021 / Published online: August 13, 2021

(C) The Author(s) 2021

\section{ABSTRACT}

Introduction: Wheelchair users are at a high risk of experiencing non-neuropathic pain of musculoskeletal origin as a result of being wheelchair-bound. The aim of this systematic

Supplementary Information The online version contains supplementary material available at https:// doi.org/10.1007/s40122-021-00294-5.

A. Liampas · P. Neophytou - C. Ioannou .

G. M. Hadjigeorgiou · P. Zis ( $\square)$

Medical School, University of Cyprus, Nicosia, Cyprus

e-mail: takiszis@gmail.com

\section{A. Liampas}

e-mail: liampasand@gmail.com

P. Neophytou

e-mail: Pana_ne@hotmail.com

C. Ioannou

e-mail: ioannou.christiana3@gmail.com

M. Sokratous · G. M. Hadjigeorgiou · P. Zis

Department of Neurology, Nicosia General Hospital, Nicosia, Cyprus

M. Sokratous

e-mail: marysokra@gmail.com

G. M. Hadjigeorgiou

e-mail: hadjigeorgiou.georgios@ucy.ac.cy

G. Varrassi

Paolo Procacci Foundation, Rome, Italy

e-mail: giuvarr@gmail.com review was to establish the prevalence of musculoskeletal pain in wheelchair users that is attributable to wheelchair use, and to describe the different pain syndromes and discuss risk factors and management options.

Methods: After a systematic MEDLINE search, we identified 40 papers eligible for inclusion.

Results: The pooled prevalence of musculoskeletal pain at any location was 50\% (95\% CI 33-67\%). The most common pain syndrome was shoulder pain (pooled prevalence $44 \%, 95 \%$ CI 36-52\%). Wheelchair users were 5.8 times as likely to suffer from shoulder pain as controls (95\% CI 2.7-12.2, $p<0.0001)$. Other pain syndromes included neck, elbow, wrist, hand and low back pain. Older age and increased duration of wheelchair use were the most significant determinants of pain in wheelchair users.

Conclusions: Musculoskeletal pain as a result of wheelchair use is very common amongst wheelchair users. Management of pain should follow national and international guidelines. Optimal adjustment of seating position may prevent pain, and is important to be taken into consideration.

Keywords: Musculoskeletal pain; Wheelchair; Risk factors; Management 


\section{Key Summary Points}

Musculoskeletal pain as a result of the wheelchair use is very common amongst wheelchair users affecting $50 \%$ of patients (95\% CI 33-67\%).

The most common pain syndrome is shoulder pain (pooled prevalence $44 \%$, 95\% CI 36-52\%).

Other pain syndromes include neck, elbow, wrist, hand and low back pain.

Optimal adjustment of seating position may prevent pain and is important to be taken into consideration.

\section{INTRODUCTION}

Mobility impairment is amongst the leading causes of disability [1]. Factors leading to mobility restriction and, subsequently, wheelchair use include chronic neurological disorders such as multiple sclerosis, stroke and spinal cord injuries. The underlying disease is often associated with neuropathic pain, due to the involvement of the somatosensory tract [2]; however, wheelchair users are at high risk of experiencing non-neuropathic pain, of musculoskeletal origin. Such musculoskeletal pain may be associated with the underlying disease (i.e. presence of spasticity) or may be a direct consequence of being wheelchair-bound.

Musculoskeletal pain imposes an extra burden on the quality of life for wheelchair users [3], and therefore it is important to prevent but also to manage it adequately when present.

The aim of this systematic review of the current literature was to establish the prevalence of musculoskeletal pain in wheelchair users that is attributable to wheelchair use, and to describe the different musculoskeletal pain syndromes and discuss risk factors and the pharmacological or non-pharmacological management options.

\section{METHODS}

\section{Protocol Registration}

This review was registered in PROSPERO, an international prospective register of systematic reviews, under registration no. CRD42020220259.

\section{Literature Search Strategy}

A systematic literature search in the PubMed database was performed on 7 November 2020 using two medical subject heading (MeSH) terms. Term A was "pain OR painful"; term B was "bedbound" OR "wheelchair" OR "restricted mobility" OR "reduced mobility" OR "limited mobility". Human subject and English language filters were applied. The reference lists of articles that met the eligibility criteria were further screened to identify additional studies that may fall within the scope of this review.

\section{Inclusion and Exclusion Criteria and Screening Process}

Articles eligible for inclusion in this review were required to meet the following criteria: (1) the article discussed musculoskeletal pain attributable to the use of a wheelchair; (2) the study was conducted using human subjects; (3) the article was written in English.

The exclusion criteria were as follows: (1) studies referring to other types of pain (i.e. neuropathic); (2) subjects were not wheelchair users; (3) pain was not clearly a result of wheelchair use (i.e. pain was linked to the injury leading to the wheelchair use rather than the wheelchair use per se); (4) assessment or management of pain was not the primary aim of the study; (5) the paper was not an original study (i.e. review articles, letters, medical hypotheses, etc.); (6) questionnaire/interviewbased surveys with no clinical evaluation of the patients by a physician; (7) papers referring to the exact same population; (8) case series/cohorts with fewer than 10 wheelchair users; (9) articles where the full text was not available. 
PRISMA 2009 Flow Diagram
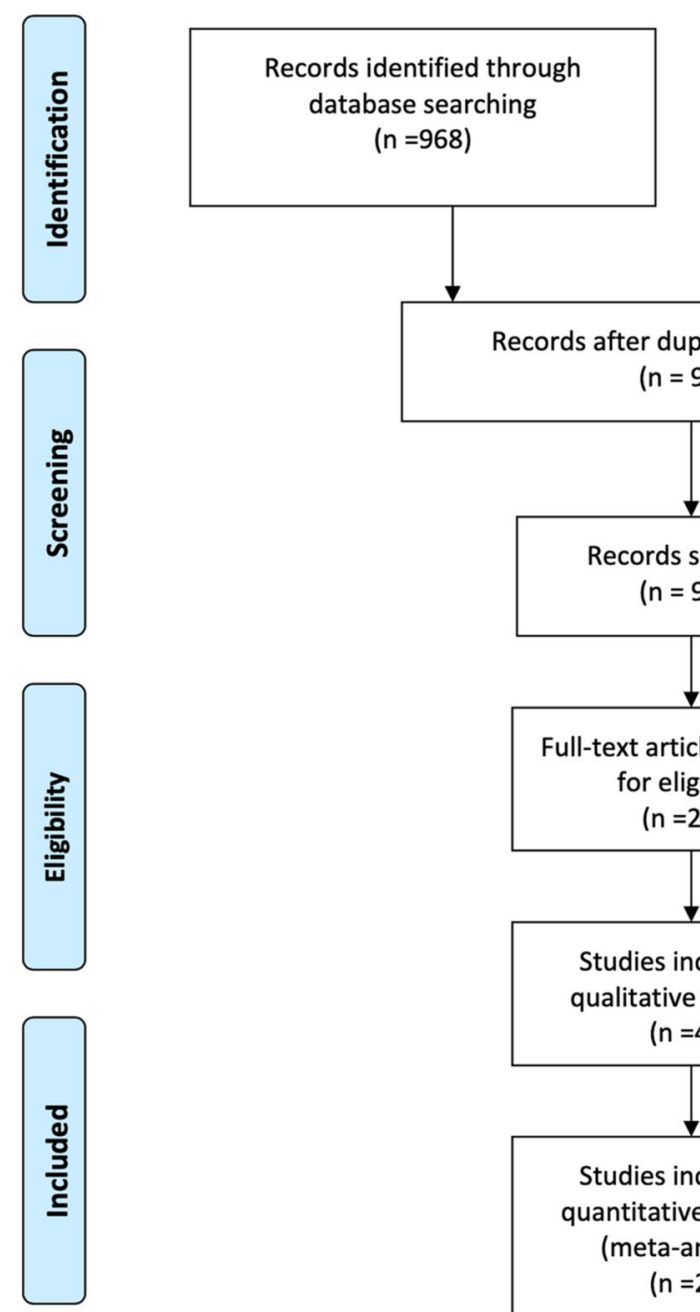

Records after duplicates removed

( $n=968)$
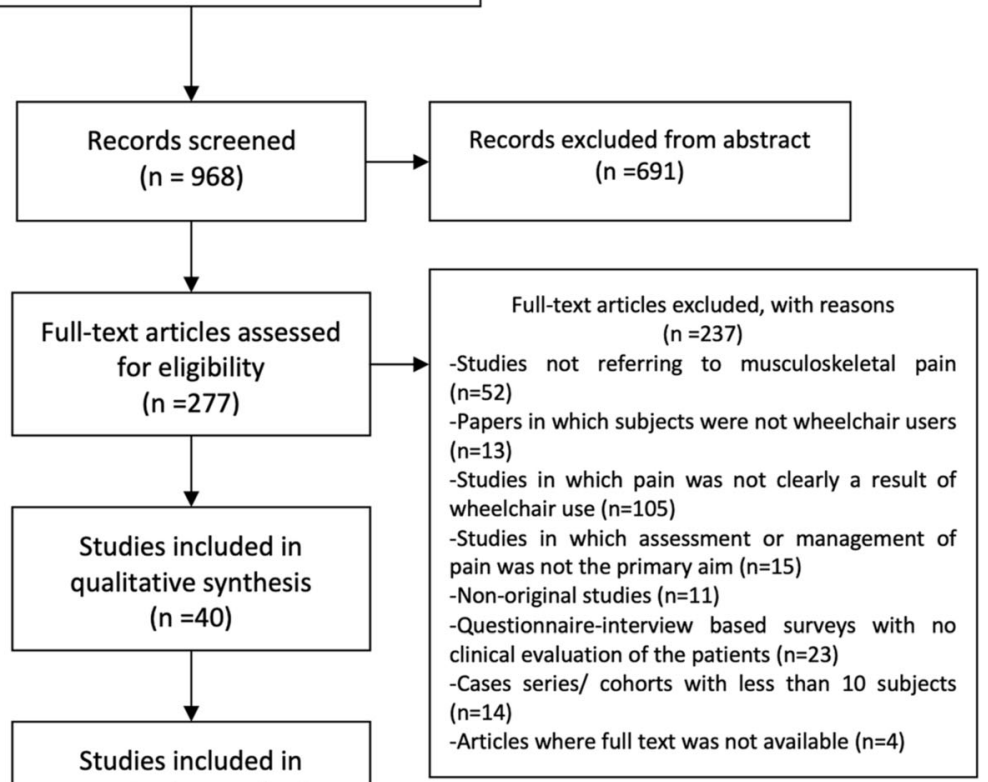

quantitative synthesis

(meta-analysis)

$(n=23)$

Fig. 1 PRISMA flow chart

All papers were screened in triplicate. Conflicts were settled by a discussion, during which a consensus was reached.

This study is reported in accordance with the Preferred Reporting Items for Systematic Reviews and Meta-Analyses (PRISMA) guidelines [4]. Details of this process are illustrated in Fig. 1.

\section{Data Collection Process}

Data were extracted from each study in a structured coding scheme using Excel. Data collected included population size, demographics, weight, BMI, prevalence, occupation, pain location, comorbidities, means of diagnosis, duration of dependency, level of injury, management, prognosis, risk factors. 


\section{Synthesis of Results}

This study used aggregate data where possible. Statistical calculation of pooled proportions was conducted in $\mathrm{R}$ language using the default settings of the "meta" package and the "metaprop" function with a random effects model [5]. The meta-analysis of odds ratios was conducted using the RevMan program [6] as suggested by the Cochrane Collaboration Group. The heterogeneity between studies was assessed by the $I^{2}$ test [7]. Often, data did not lend itself to meta-analysis and therefore a narrative approach was taken.

\section{Quality Assessment of Included Studies}

We evaluated the methodological quality of prevalence studies using a checklist adapted from Hoy et al. [8]. It consists of nine questions that assess the representativeness of the sample, the sampling technique, the response rate, the data collection method, the measurement tools, the case definitions and the statistical reporting. Each checked question was scored as either " 0 " or " 1 ", corresponding respectively to "low risk of bias" and "high risk of bias". The total score ranged from 0 to 9 , with the overall score categorized as follows: 7-9: "high risk of bias", 4-6: "moderate risk", and 0-3: "low risk" [2]. For management studies, we used the Cochrane Collaboration's tool for assessing the risk of bias in randomized trials [9] and the Newcastle-Ottawa scale, which is approved by the Cochrane Collaboration, for assessing the quality of nonrandomized studies [10]. The evaluation of all included studies is available as supplementary material.

\section{Compliance with Ethical Guidelines}

The article is based on previously conducted studies. Thus, there were no ethical concerns with respect to this study, nor was approval of the research protocol from an ethics committee required.

\section{RESULTS}

\section{Study Characteristics}

Forty studies met the above-mentioned inclusion criteria. Of those studies, 32 were of an observational nature: 20 case series/cross-sectional studies, seven case-control studies and five cohort studies. Eight were interventional studies: four randomized clinical trials (RCT) and four open-label trials.

\section{Prevalence}

Twenty-three studies investigated non-selected populations of wheelchair users for musculoskeletal pain [11-33]. Of those, only two provided data for the presence of musculoskeletal pain at any location, whereas the rest provided data for the presence of specific pain syndromes, namely shoulder pain $(n=20)$, elbow pain $(n=4)$, wrist pain $(n=4)$, neck pain $(n=3)$, hand pain $(n=3)$, low back pain $(n=2)$, thoracic pain $(n=1)$ and forearm pain $(n=1)$.

Figure 2 shows the pooled prevalence of musculoskeletal pain at any location in wheelchair users, following the meta-analysis of two available studies $[11,12]$. The pooled prevalence was 50\% (95\% CI 33-67\%). However, there was substantial heterogeneity across the included studies $\left(I^{2}=77 \%\right)$.

Figure 3 shows the pooled prevalence of shoulder pain in wheelchair users, following the meta-analysis of 20 available studies [11-30]. The pooled prevalence was $44 \% \quad(95 \%$ CI $36-52 \%)$. However, there was substantial heterogeneity across the included studies $\left(I^{2}=85 \%\right)$. The meta-analysis of five case-control studies is summarized in Fig. 4; the odds of experiencing shoulder pain were significantly higher in wheelchair user groups than in controls (OR 5.8, 95\% CI 2.7-12.2, $p<0.0001$ ).

Figure 5 shows the pooled prevalence of elbow pain in wheelchair users, following the meta-analysis of four available studies $[11,12,25,29]$. The pooled prevalence was $21 \%$ (95\% CI 10-38\%). However, there was substantial heterogeneity across the included studies $\left(I^{2}=74 \%\right)$. 


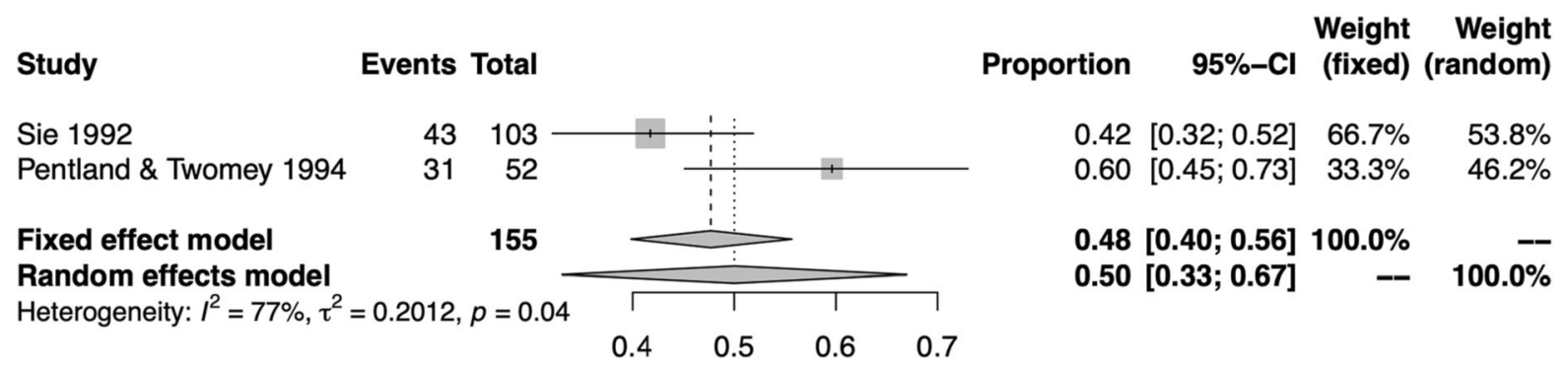

Fig. 2 Forest plot of the prevalence of musculoskeletal pain at any location amongst wheelchair users

\section{Study}

Ortega-santiago 2020

Rice 2014

Giner-Pascual 2011

Nawoczenski 2006

Collinger 2008

Mercer 2006

Akbar 2011

Mulroy 2015

Gil-Agudo 2015

Collinger 2010

Troy 2014

Forslund 2007

Norrbrink 2012

Silfverskiold \& Waters 1991

Sie 1992

Ballinger 2000

Escobedo 1997

Pentland \& Twomey 1991

Pentland \& Twomey 1994

Bayley 1987

Fixed effect model

Random effects model

Heterogeneity: $I^{2}=85 \%, \tau^{2}=0.3968, p<0.01$
Events Total

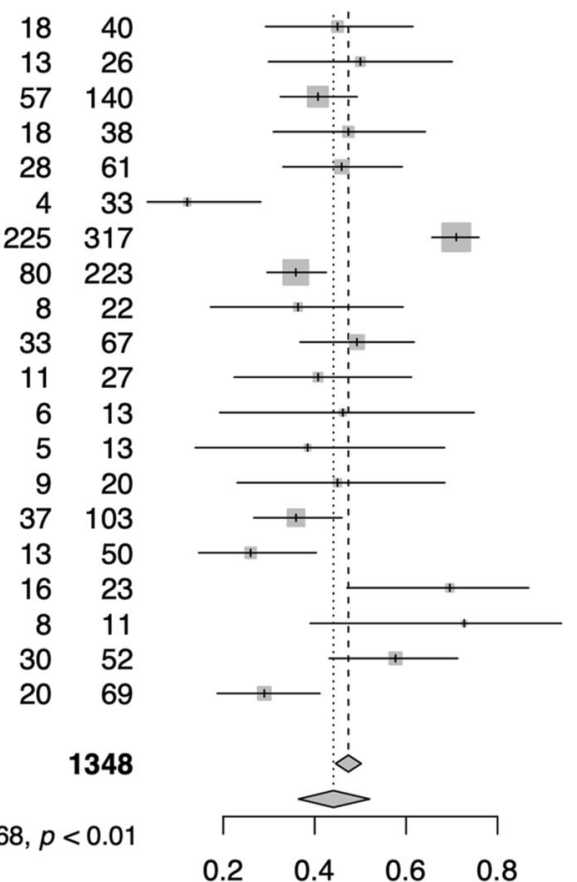

Proportion $95 \%$

$0.45[0.29 ; 0.62] \quad 3.3 \% \quad 5.3 \%$

$0.50[0.30 ; 0.70] \quad 2.2 \% \quad 4.8 \%$

$0.41[0.32 ; 0.49] \quad 11.2 \% \quad 6.2 \%$

$0.47[0.31 ; 0.64] \quad 3.1 \% \quad 5.2 \%$

$0.46[0.33 ; 0.59] \quad 5.0 \% \quad 5.7 \%$

$0.12[0.03 ; 0.28] \quad 1.2 \% \quad 3.8 \%$

$0.71[0.66 ; 0.76] \quad 21.6 \% \quad 6.4 \%$

$0.36[0.30 ; 0.43] \quad 17.0 \% \quad 6.3 \%$

$0.36[0.17 ; 0.59] \quad 1.7 \% \quad 4.4 \%$

$0.49[0.37 ; 0.62] \quad 5.5 \% \quad 5.7 \%$

$0.41[0.22 ; 0.61] \quad 2.2 \% \quad 4.8 \%$

$0.46[0.19 ; 0.75] \quad 1.1 \% \quad 3.7 \%$

$0.38[0.14 ; 0.68] \quad 1.0 \% \quad 3.6 \%$

$0.45[0.23 ; 0.68] \quad 1.6 \% \quad 4.4 \%$

$0.36[0.27 ; 0.46] \quad 7.9 \% \quad 6.0 \%$

$0.26[0.15 ; 0.40] \quad 3.2 \% \quad 5.2 \%$

$0.70[0.47 ; 0.87] \quad 1.6 \% \quad 4.4 \%$

$0.73[0.39 ; 0.94] \quad 0.7 \% \quad 3.1 \%$

$0.58[0.43 ; 0.71] \quad 4.2 \% \quad 5.5 \%$

$0.29[0.19 ; 0.41] \quad 4.7 \% \quad 5.6 \%$

$\begin{array}{llr}0.47[0.45 ; 0.50] & 100.0 \% & -- \\ 0.44[0.36 ; 0.52] & -- & 100.0 \%\end{array}$

Fig. 3 Forest plot of the prevalence of shoulder musculoskeletal pain amongst wheelchair users

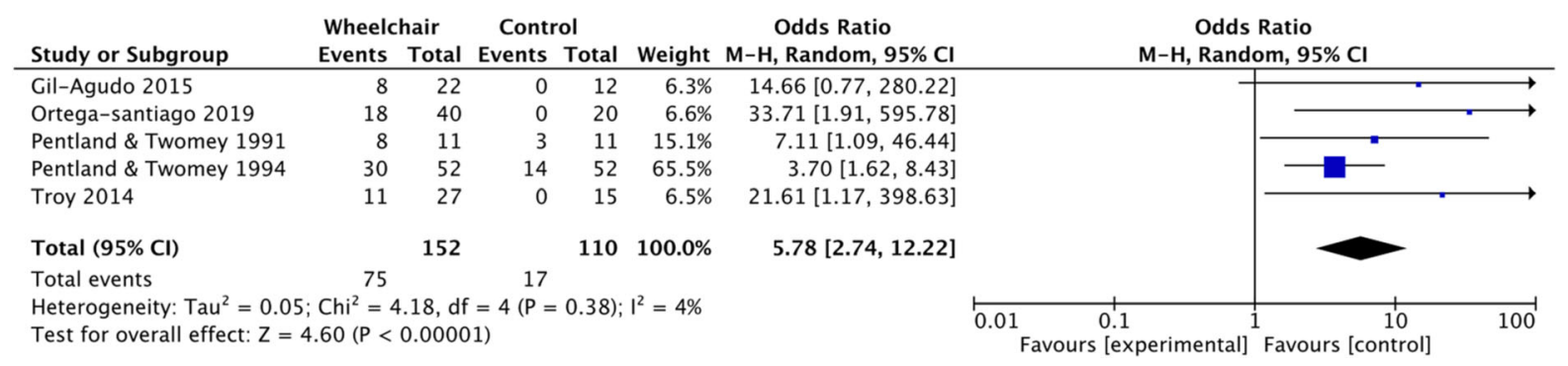

Fig. 4 Meta-analysis results as illustrated in the forest plot regarding the odds of having shoulder pain in wheelchair users compared to controls 


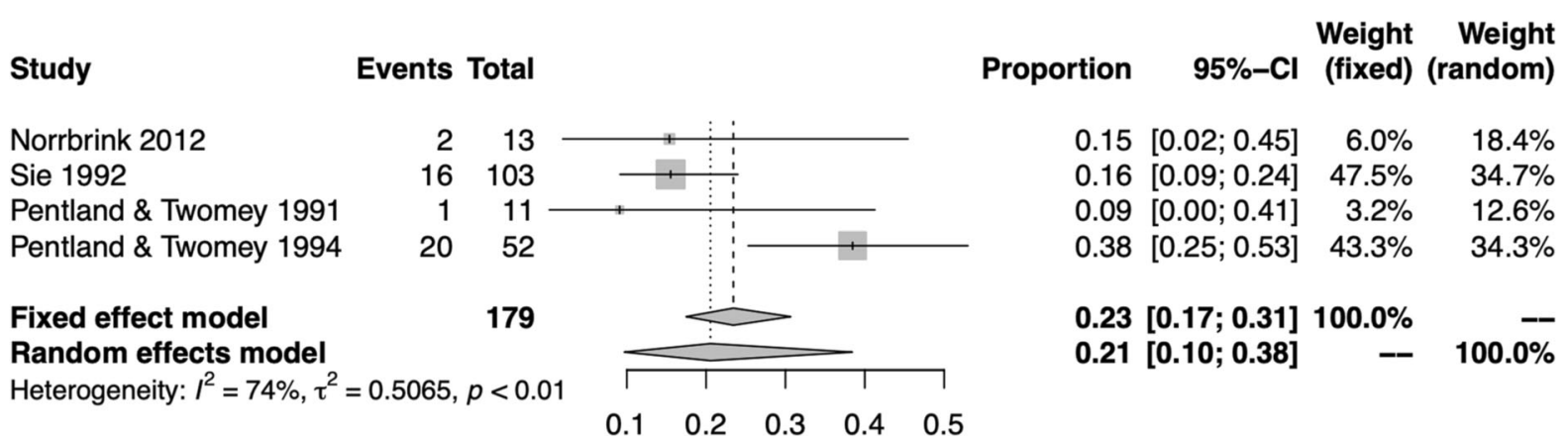

Fig. 5 Forest plot of the prevalence of elbow musculoskeletal pain amongst wheelchair users

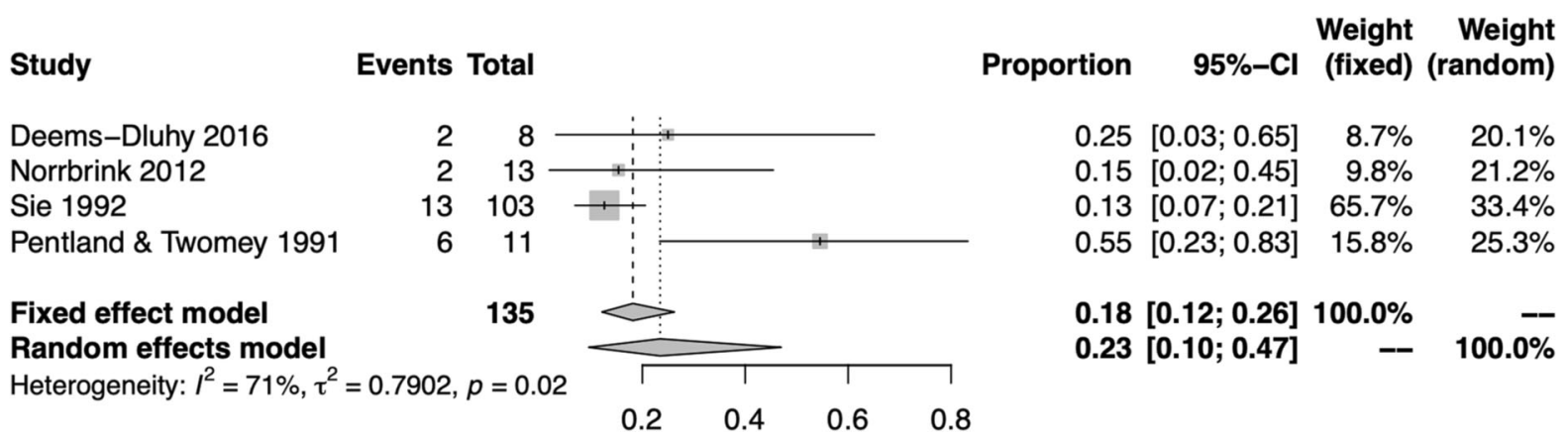

Fig. 6 Forest plot of the prevalence of wrist musculoskeletal pain amongst wheelchair users

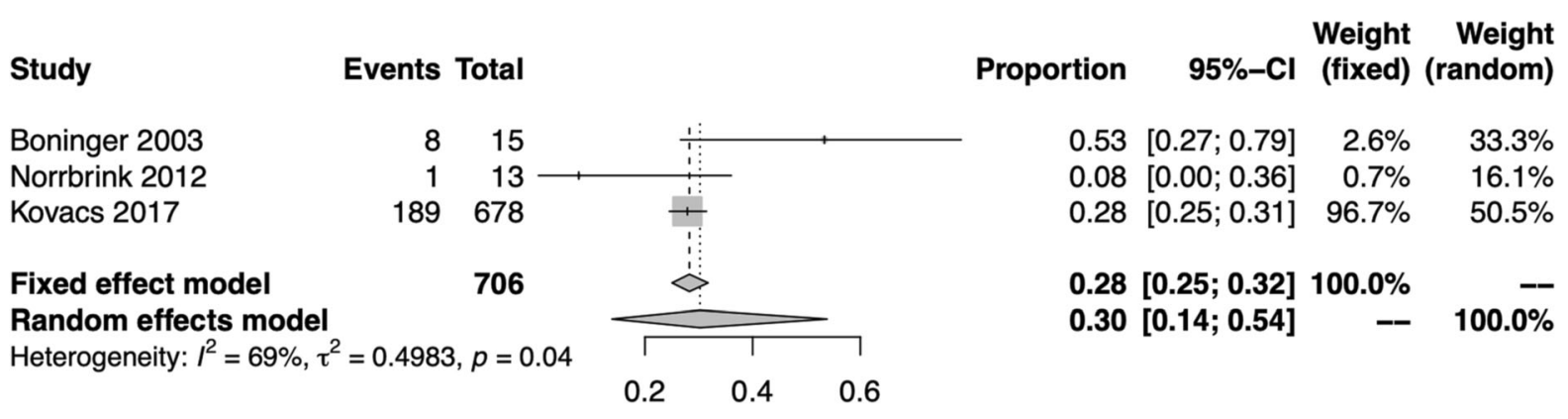

Fig. 7 Forest plot of the prevalence of neck musculoskeletal pain amongst wheelchair users

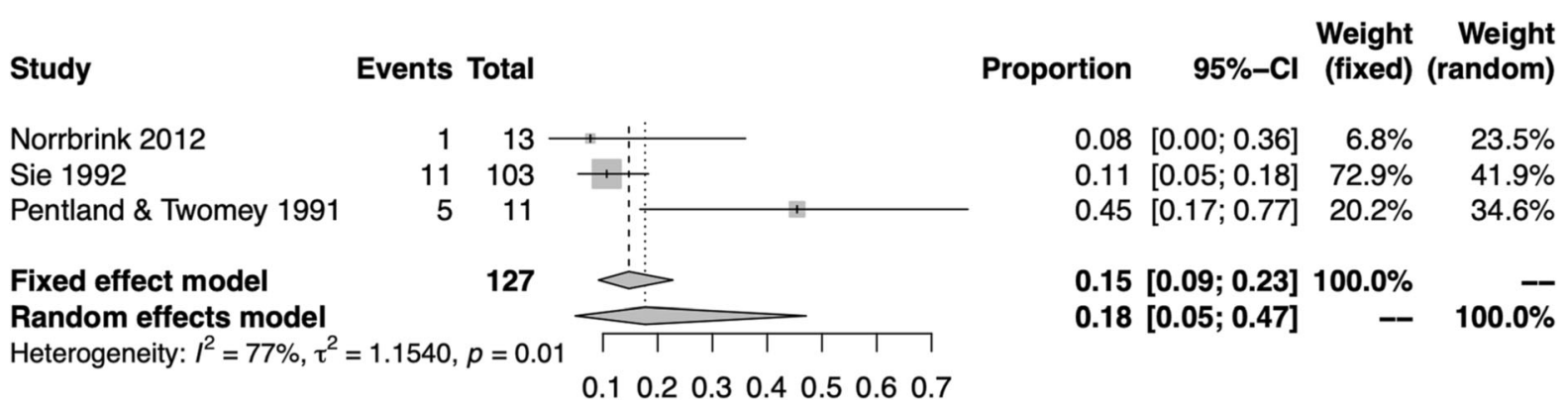

Fig. 8 Forest plot of the prevalence of hand musculoskeletal pain amongst wheelchair users 


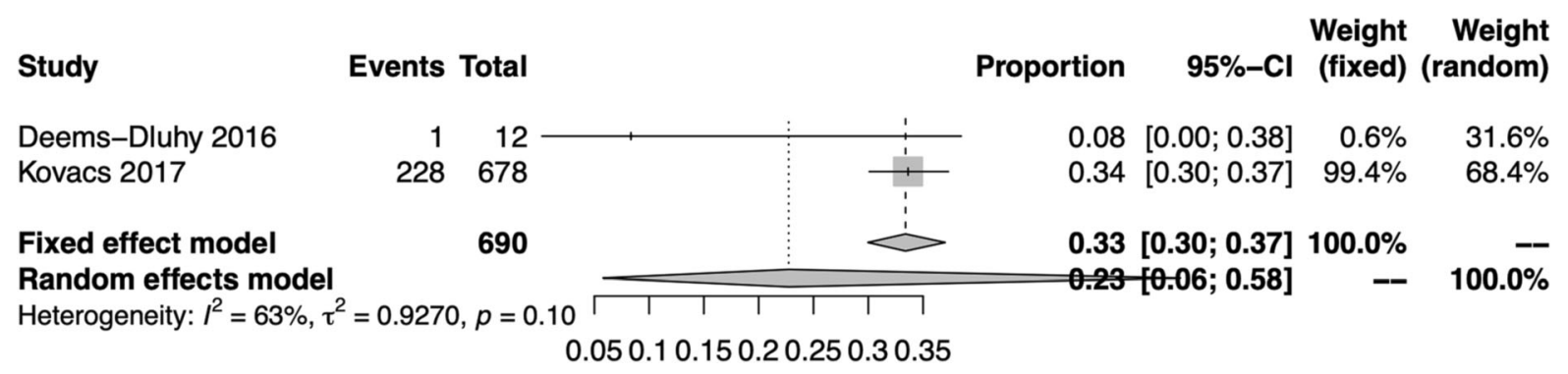

Fig. 9 Forest plot of the prevalence of low back musculoskeletal pain amongst wheelchair users

Figure 6 shows the pooled prevalence of wrist pain in wheelchair users, following the meta-analysis of four available studies $[11,25,29,31]$. The pooled prevalence was $23 \%$ (95\% CI 10-47\%). However, there was substantial heterogeneity across the included studies $\left(I^{2}=71 \%\right)$.

Figure 7 shows the pooled prevalence of neck pain in wheelchair users, following the metaanalysis of three available studies [25, 32, 33]. The pooled prevalence was 30\% (95\% CI 14-54\%). However, there was substantial heterogeneity across the included studies $\left(I^{2}=69 \%\right)$.

Figure 8 shows the pooled prevalence of hand pain in wheelchair users, following the meta-analysis of three available studies $[11,25,29]$. The pooled prevalence was $18 \%$ (95\% CI 5-47\%). However, there was substantial heterogeneity across the included studies $\left(I^{2}=77 \%\right)$.

Figure 9 shows the pooled prevalence of low back pain in wheelchair users, following the meta-analysis of two available studies [31, 33]. The pooled prevalence was $23 \% \quad(95 \%$ CI 6-58\%). However, there was substantial heterogeneity across the included studies $\left(I^{2}=63 \%\right)$.

Single studies reported that the prevalence of thoracic [33] and forearm [25] musculoskeletal pain was $20 \%$ and $15 \%$, respectively.

\section{Types of Musculoskeletal Pain}

Shoulder pain: the most frequently reported type of musculoskeletal pain in wheelchair users is mainly related to rotator cuff disorders [34]. Possible pathogenetic mechanisms include joint inflammation [35], rotator cuff tears and subsequent instability of the glenohumeral joint $[15,19,28,30,36]$ and tendinopathies $[22,26,37,38]$. Shoulder impingement syndrome $[11,26,30]$ mostly occurs due to upperextremity propulsion forces [18] and weightbearing tasks [16, 17, 30, 38]. Furthermore, instability of the joint [37], adductor muscle weakness [39], chronic overuse [11, 19] and repetitive traumas have all been implicated in the pathogenesis of shoulder pain [15, 40, 41]. Shoulder pain has a significant effect on the range of motion (ROM), leading to functional limitations [27, 42, 43].

Elbow [11, 12, 25, 29], forearm [25], wrist $[11,25,29,31]$ and hand pain $[11,25,29]$ occurs as a result of overuse during propulsion.

Neck, thoracic and back pain occurs as a result of poor posture [33, 44, 45]. Boninger et al. suggested that neck pain could be of mechanical or myofascial origin [32].

\section{Risk Factors}

Risk factors are widely commented upon in the literature, largely on the basis of epidemiological studies.

Age

A few studies showed that increasing age is a risk factor for shoulder pain $[22,28]$ in wheelchair users. Tsunoda et al. highlighted that older age is mainly associated with greater shoulder pain in men [35]. Akbar et al. suggested that a possible explanation for this finding is that rotator cuff tears occur more frequently in older wheelchair users [19]. 


\section{Duration of Wheelchair Use}

Increased duration since injury and, subsequently, increased duration of wheelchair use is a risk factor for upper extremity pain in wheelchair users [12, 22, 27, 28, 37]. Akbar et al. suggested that a longer period of wheelchair dependency increases the risk of rotator cuff tears [19].

\section{Sex}

Current evidence seems to be inconsistent regarding sex as a risk factor. Two studies support an increased risk of upper extremity pain in females, as the greater ROM in female subjects could predispose them to injury [29, 42]. Tsunoda et al., however, suggested the opposite, that male wheelchair users are more likely to experience shoulder pain than females due to lower shoulder flexibility [35].

\section{Body Mass Index}

In an ultrasound examination-based study of the non-dominant shoulder in manual wheelchair users, Collinger et al. found that increased body mass index (BMI) is associated with tendon degeneration and represents a risk factor of shoulder pain [22]. Increased body weight can lead to rotator cuff enthesis, acromioclavicular joint degeneration, distal clavicular osteolysis and glenohumeral joint loading, which in turn may increase the risk for shoulder injury $[17,46]$. However, although most studies found a link between increased BMI and musculoskeletal pain, in their case-control study, Akbar et al. failed to show such a link in a large group of paraplegic patients [47].

\section{Level of Spinal Cord Injury (SCI)}

In a cross-sectional study, Sinnott et al. argued that patients with SCI at a higher level are more likely to suffer from rotator cuff disorders [34]; however, this was not confirmed in a more recent and larger case-control study conducted by Akbar et al. [47].

\section{The Role of Activity}

There is conflicting evidence with regard to the relationship between activity and the risk of musculoskeletal pain in wheelchair users.
Ortega-Santiago et al. found a positive relationship between activity and musculoskeletal pain [13]. The authors argued that the main cause is the overuse of the upper extremities. Akbar et al. suggested that recurrent over-thehead shoulder movement from a wheelchair position predisposes to the development of shoulder pathology in paraplegics [47]. Activity, however, correlates with age, as younger individuals tend to be more active. In their study, Collinger et al. suggested that younger people experienced shoulder pain during propulsion more frequently because of the increased activity [17]. On the contrary, some studies did not find that daily physical activity [20] or involvement in sports [37] was associated with the development of shoulder pain.

\section{Management}

Management of musculoskeletal pain in wheelchair users follows the national and international guidelines for the management of pain [48]. However, a few studies have tried specific interventions, pharmacological or nonpharmacological, in wheelchair users with musculoskeletal pain and are discussed below.

\section{Pharmacological}

Giner-Pascual et al. conducted a 6-month double-blind, placebo-controlled RCT and found that the transdermal nitroglycerine patch $(1.25$ $\mathrm{mg}$ ) as monotherapy was safe and effective in alleviating pain and improved the function and range of the articular shoulder [49].

\section{Exercise}

In their RCT, Curtis et al. showed that a combination of adjuvant stretching and strengthening exercises decreased pain intensity in subjects with shoulder pain, but the benefit was not statistically significant [41]. Nawoczenski et al. conducted a controlled selective 8-week interventional study in wheelchair users with SCI and shoulder impingement symptoms and showed that exercise was effective in alleviating pain and improving function and satisfaction in these subjects [16]. In a 12-week single-blind RCT, Mulroy et al. demonstrated that a home- 
based movement optimization intervention provided pain relief and improvement in muscle strength and overall quality of life, an effect which persisted for at least 4 weeks post-intervention [50].

\section{Functional Electrical Stimulation}

In a 6-week open-label study, Wilbanks et al. studied the efficacy of functional electrical stimulation-assisted rowing in subjects with SCI and found that it was effective in improving aerobic fitness and pain [51].

\section{Seating Position Adjustment}

Uncomfortable seating and bad posture have been linked to musculoskeletal pain in wheelchair users. Samuelsson et al. suggest that the exchange of the wheelchair into a more adjustable one with better ergonomic settings can improve low back pain [44].

Regarding neck pain, neck flexion and rotation [45] increase neck discomfort in wheelchair users and seem to contribute to the development of neck pain. Kirby et al. suggested that the most comfortable position for most wheelchair users is straight ahead with the neck slightly flexed [45]. Furthermore, the thickness of the wheelchair seat cushion has been proposed as a modifiable risk factor for the development of neck pain [33].

Regarding shoulder pain, Giner-Pascual et al. found that a straight position of the seat rather than an angled position may be a risk factor for shoulder injury and subsequent pain [15].

\section{CONCLUSIONS}

Using a meta-analytic approach, we established the prevalence of musculoskeletal pain as a result of wheelchair use and showed that one in two wheelchair users will experience such pain. Shoulder pain is the most frequent type of musculoskeletal pain syndrome.

Amongst the predictors of musculoskeletal pain, older age and increased duration of wheelchair use seem to play a significant role. Evidence of other risk factors such as sex, BMI and degree of activity remains conflicting, and thus larger studies which will allow for multivariate analyses are needed.

Management of musculoskeletal pain should follow national and international guidelines. However, it is evident that optimal adjustment of seating position may prevent pain and is important to be taken into consideration.

Our results should be interpreted with some caution given the limitations of our design. Firstly, there was a great deal of heterogeneity between studies used in the meta-analysis. Secondly, we only searched for publications in PubMed and, therefore, might have missed a few more papers that are indexed only in other databases. Thirdly, there were no restrictions in date of publication applied in this review. This was a deliberate decision made in order to review the full range of literature pertinent to the topic in question. However, a consequence of inclusion of older literature is heterogeneity, as the clinical outcomes changed over time. Finally, we excluded papers reporting upper limb pain in patients with tetraplegia, as we wanted to be sure that the pain was not a result of the underlying injury. Therefore, our prevalence figures do not necessarily depict the prevalence of musculoskeletal pain in tetraplegic wheelchair users.

\section{ACKNOWLEDGEMENTS}

Funding. No funding and/or sponsorship was received in relation to this paper.

Authorship. All named authors meet the International Committee of Medical Journal Editors (ICMJE) criteria for authorship for this article, take responsibility for the integrity of the work as a whole, and have given their approval for this version to be published.

Authors' Contribution. AL: study design, data extraction, statistical analysis, manuscript drafting. PN: data extraction, manuscript revision. MS: data extraction, manuscript revision. GMH: study design, manuscript revision. GV: study design, manuscript revision. CI: data extraction, manuscript revision, linguistic 
revision. PZ: study design, data extraction, statistical analysis, manuscript revision.

Disclosures. Andreas Liampas, Panayiota Neophytou, Maria Sokratous, Christiana Ioannou, and Georgios M. Hadjigeorgiou have nothing to disclose. Giustino Varrassi is the journal's Editor and Panagiotis Zis is a member of the journal's Editorial Board.

Compliance with Ethics Guidelines. This article is based on previously conducted studies and does not contain any new studies with human participants or animals performed by any of the authors.

Data Availability. The datasets analyzed during the current study are available from the corresponding author upon reasonable request.

Open Access. This article is licensed under a Creative Commons Attribution-NonCommercial 4.0 International License, which permits any non-commercial use, sharing, adaptation, distribution and reproduction in any medium or format, as long as you give appropriate credit to the original author(s) and the source, provide a link to the Creative Commons licence, and indicate if changes were made. The images or other third party material in this article are included in the article's Creative Commons licence, unless indicated otherwise in a credit line to the material. If material is not included in the article's Creative Commons licence and your intended use is not permitted by statutory regulation or exceeds the permitted use, you will need to obtain permission directly from the copyright holder. To view a copy of this licence, visit http://creativecommons.org/licenses/by$\mathrm{nc} / 4.0 /$.

\section{REFERENCES}

1. Smith EM, Giesbrecht EM, Mortenson WB, et al. Prevalence of wheelchair and scooter use among community-dwelling Canadians. Phys Ther. 2016;96(8):1135-42.
2. Liampas A, Velidakis N, Georgiou T, et al. Prevalence and management challenges in central poststroke neuropathic pain: a systematic review and meta-analysis. Adv Ther. 2020;37(7):3278-91.

3. Gutierrez DD, Thompson L, Kemp B, et al. The relationship of shoulder pain intensity to quality of life, physical activity, and community participation in persons with paraplegia. J Spinal Cord Med. 2007;30(3):251-5.

4. Moher D, Liberati A, Tetzlaff J, et al. Preferred reporting items for systematic reviews and metaanalyses: the PRISMA statement. PLoS Med. 2009;6(7):e1000097.

5. Langfelder P, Horvath S. WGCNA: an R package for weighted correlation network analysis. BMC Bioinform. 2008;9:559-559.

6. Review Manager (RevMan) [Computer program]. Version 5.4. Copenhagen: The Nordic Cochrane Centre, The Cochrane Collaboration, 2020.

7. Higgins JP, Thompson SG. Quantifying heterogeneity in a meta-analysis. Stat Med. 2002;21: 1539-58.

8. Hoy D, Brooks P, Woolf A, et al. Assessing risk of bias in prevalence studies: modification of an existing tool and evidence of interrater agreement. J Clin Epidemiol. 2012;65:934-9.

9. Higgins JP, Altman DG, Gøtzsche PC, et al. The Cochrane Collaboration's tool for assessing risk of bias in randomised trials. BMJ. 2011;18(343):d5928.

10. Wells GA, Shea B, O'Connell D et al. The Newcastle-Ottawa Scale (NOS) for assessing the quality of nonrandomised studies in meta-analyses, http:// www.ohri.ca/programs/clinical_epidemiology/ oxford.asp. (2008, Assessed 5 Jan 2021)

11. Sie IH, Waters, Adkins RH, et al. Upper extremity pain in the postrehabilitation spinal cord injured patient. Arch Phys Med Rehabil. 1992;73(1):44-8.

12. Pentland WE, Twomey LT. Upper limb function in persons with long term paraplegia and implications for independence: part I. Paraplegia. 1994;32(4): 211-8.

13. Ortega-Santiago R, González-Aguado ÁJ, Fernándezde-Las-Peñas C, et al. Pressure pain hypersensitivity and referred pain from muscle trigger points in elite male wheelchair basketball players. Braz J Phys Ther. 2020;24(4):333-41.

14. Rice IM, Jayaraman C, Hsiao-Wecksler ET, et al. Relationship between shoulder pain and kinetic and temporal-spatial variability in wheelchair users. Arch Phys Med Rehabil. 2014;95(4):699-704. 
15. Giner-Pascual M, Alcanyis-Alberola M, González LM, et al. Shoulder pain in cases of spinal injury: influence of the position of the wheelchair seat. Int J Rehabil Res. 2011;34(4):282-9.

16. Nawoczenski DA, Ritter-Soronen JM, Wilson CM, et al. Clinical trial of exercise for shoulder pain in chronic spinal injury. Phys Ther. 2006;86(12): 1604-18.

17. Collinger JL, Boninger ML, Koontz AM, et al. Shoulder biomechanics during the push phase of wheelchair propulsion: a multisite study of persons with paraplegia. Arch Phys Med Rehabil. 2008;89(4):667-76.

18. Mercer JL, Boninger M, Koontz A, et al. Shoulder joint kinetics and pathology in manual wheelchair users. Clin Biomech (Bristol, Avon). 2006;21(8): 781-9.

19. Akbar M, Brunner M, Balean G, et al. A cross-sectional study of demographic and morphologic features of rotator cuff disease in paraplegic patients. J Shoulder Elbow Surg. 2011;20(7):1108-13.

20. Mulroy SJ, Hatchett P, Eberly VJ, et al. Shoulder strength and physical activity predictors of shoulder pain in people with paraplegia from spinal injury: prospective cohort study. Phys Ther. 2015;95(7):1027-38.

21. Gil-Agudo A, Mozos MS, Crespo-Ruiz B, et al. Shoulder kinetics and ultrasonography changes after performing a high-intensity task in spinal cord injury subjects and healthy controls. Spinal Cord. 2016;54(4):277-82.

22. Collinger JL, Fullerton B, Impink BG, et al. Validation of grayscale-based quantitative ultrasound in manual wheelchair users: relationship to established clinical measures of shoulder pathology. Am J Phys Med Rehabil. 2010;89(5):390-400.

23. Troy KL, Munce TA, Longworth JA. An exercise trial targeting posterior shoulder strength in manual wheelchair users: pilot results and lessons learned. Disabil Rehabil Assist Technol. 2015;10(5):415-20.

24. Forslund EB, Granström A, Levi R, et al. Transfer from table to wheelchair in men and women with spinal cord injury: coordination of body movement and arm forces. Spinal Cord. 2007;45(1):41-8.

25. Norrbrink C, Lindberg T, Wahman K, et al. Effects of an exercise programme on musculoskeletal and neuropathic pain after spinal cord injury-results from a seated double-poling ergometer study. Spinal Cord. 2012;50(6):457-61.
26. Silfverskiold J, Waters RL. Shoulder pain and functional disability in spinal cord injury patients. Clin Orthop Relat Res. 1991;272:141-5.

27. Ballinger DA, Rintala DH, Hart KA. The relation of shoulder pain and range-of-motion problems to functional limitations, disability, and perceived health of men with spinal cord injury: a multifaceted longitudinal study. Arch Phys Med Rehabil. 2000;81(12):1575-81.

28. Escobedo EM, Hunter JC, Hollister MC, et al. MR imaging of rotator cuff tears in individuals with paraplegia. AJR Am J Roentgenol. 1997;168(4): 919-23.

29. Pentland WE, Twomey LT. The weight-bearing upper extremity in women with long term paraplegia. Paraplegia. 1991;29(8):521-30.

30. Bayley JC, Cochran TP, Sledge CB. The weightbearing shoulder. The impingement syndrome in paraplegics. J Bone Jt Surg Am. 1987;69(5):676-8.

31. Deems-Dluhy SL, Jayaraman C, Green S, et al. Evaluating the functionality and usability of two novel wheelchair anti-rollback devices for ramp ascent in manual wheelchair users with spinal cord injury. PM R. 2017;9(5):483-93.

32. Boninger ML, Cooper RA, Fitzgerald SG, et al. Investigating neck pain in wheelchair users. Am J Phys Med Rehabil. 2003;82(3):197-202.

33. Kovacs FM, Seco L, Royuela A, et al. Prevalence and factors associated with a higher risk of neck and back pain among permanent wheelchair users: a cross-sectional study. Spinal Cord. 2018;56(4): 392-405.

34. Sinnott KA, Milburn P, McNaughton H. Factors associated with thoracic spinal cord injury, lesion level and rotator cuff disorders. Spinal Cord. 2000;38(12):748-53.

35. Tsunoda K, Mutsuzaki H, Hotta K, et al. Correlates of shoulder pain in wheelchair basketball players from the Japanese national team: a cross-sectional study. J Back Musculoskelet Rehabil. 2016;29(4): 795-800.

36. Morrow MMB, Van Straaten MG, Murthy NS, et al. Detailed shoulder MRI findings in manual wheelchair users with shoulder pain. Biomed Res Int. 2014;2014:769649.

37. Finley MA, Rodgers MM. Prevalence and identification of shoulder pathology in athletic and nonathletic wheelchair users with shoulder pain: a 
pilot study. J Rehabil Res Dev. 2004;41(3B): 395-402.

38. Samuelsson KAM, Tropp H, Gerdle B. Shoulder pain and its consequences in paraplegic spinal cord-injured, wheelchair users. Spinal Cord. 2004;42(1): 41-6.

39. Walford SL, Requejo PS, Mulroy SJ, et al. Predictors of shoulder pain in manual wheelchair users. Clin Biomech (Bristol, Avon). 2019;65:1-12.

40. Burnham RS, May L, Nelson E, et al. Shoulder pain in wheelchair athletes. The role of muscle imbalance. Am J Sports Med. 1993;21(2):238-42.

41. Curtis KA, Tyner TM, Zachary L, et al. Effect of a standard exercise protocol on shoulder pain in long-term wheelchair users. Spinal Cord. 1999;37(6):421-9.

42. Wessels KW, Brown JL, Ebersole KT, et al. Sex, shoulder pain, and range of motion in manual wheelchair users. J Rehabil Res Dev. 2013;50(3): 351-6.

43. Mason BS, Vegter RJK, Paulson TAW, et al. Bilateral scapular kinematics, asymmetries and shoulder pain in wheelchair athletes. Gait Posture. 2018;65: 151-6.

44. Samuelsson K, Larsson $H$, Thyberg $M$, et al. Wheelchair seating intervention. Results from a client-centred approach. Disabil Rehabil. 2001;23(15):677-82.
45. Kirby RL, Fahie CL, Smith C, et al. Neck discomfort of wheelchair users: effect of neck position. Disabil Rehabil. 2004;26(1):9-15.

46. Boninger ML, Towers JD, Cooper RA, et al. Shoulder imaging abnormalities in individuals with paraplegia. J Rehabil Res Dev. 2001;38(4):401-8.

47. Akbar M, Balean G, Brunner M, et al. Prevalence of rotator cuff tear in paraplegic patients compared with controls. J Bone Jt Surg Am. 2010;92(1):23-30.

48. Babatunde OO, Jordan JL, Van der Windt DA, et al. Effective treatment options for musculoskeletal pain in primary care: a systematic overview of current evidence. PLoS ONE. 2017;12(6):e0178621.

49. Giner-Pascual M, Alcanyis-Alberola M, Querol F, et al. Transdermal nitroglycerine treatment of shoulder tendinopathies in patients with spinal cord injuries. Spinal Cord. 2011;49(9):1014-9.

50. Mulroy SJ, Thompson L, Kemp B, et al. Strengthening and optimal movements for painful shoulders (STOMPS) in chronic spinal cord injury: a randomized controlled trial. Phys Ther. 2011;91(3): $305-24$.

51. Wilbanks SR, Rogers R, Pool S, et al. Effects of functional electrical stimulation assisted rowing on aerobic fitness and shoulder pain in manual wheelchair users with spinal cord injury. J Spinal Cord Med. 2016;39(6):645-54. 